\title{
二溴卡宾与乙醛 $\mathbf{C}-\mathbf{H}$ 键插入反应的密度泛函理论计算
}

李志锋 ${ }^{\circledR}$, 杨声 ${ }^{\circledR}$, 吕玲玲 ${ }^{\circledR}$, 卢小泉 ${ }^{(2)}$ 康敬万 ${ }^{\circledR}$

(1) 天水师范学院生命科学与化学学院, 天水 741001 ;

(2) 西北师范大学化学化工学院, 甘肃省高分子材料重点实验室, 兰州 730070

E-mail: sciresli@163.com

2007-06-03 收稿, 2007-10-24 接受

甘肃省教育厅科研基金(批准号: 0708-11)和天水师范学院科研基金(批准号: TSA0604)资助项目

摘要 采用密度泛函理论在 B3LYP/6-31G(d)水平上研究了卡宾 $\mathrm{CH}_{2}$ 及二溴卡宾 $\mathrm{CBr}_{2}$ 与 乙醛 $\mathrm{CH}_{3} \mathrm{CHO}$ 中 $\mathrm{C}-\mathrm{H}$ 键的插入反应机理, 用频率分析和内毫反应坐标法(IRC)对过渡态 进行了验证, 计算了各物种的 $\operatorname{CCSD}(\mathrm{T}) / 6-31 \mathrm{G}(\mathrm{d})$ 及 $\operatorname{CCSD}(\mathrm{T}) / 6-31 \mathrm{G}(\mathrm{d}, \mathrm{p})$ 单点能量. 结果 表明, $\mathrm{CH}_{2}$ 与 $\mathrm{CH}_{3} \mathrm{CHO}$ 插入反应主产物为丙醛 $\left({ }^{\mathrm{H}} \mathrm{P} 1\right)$, 而 $\mathrm{CBr}_{2}$ 与 $\mathrm{CH}_{3} \mathrm{CHO}$ 插入反应的两条 主反应通道都在单重态势能面中, 单重态 $\mathrm{CBr}_{2}$ 既可以发生与 $\mathrm{C}_{\alpha}-\mathrm{H}$ 键的插入反应 I (1), 也可以发生与 $\mathrm{C}_{\beta}-\mathrm{H}$ 键插入的反应 II (1). 用经 Wigner 校正的 Eyring 过渡态理论分别计 算了反应 I (1)及 II (1)在 100 2200 K 温度范围内的热力学与动力学性质. 在 $101325 \mathrm{~Pa}$, 反应 I (1)及 II (1)进行的适宜温度范围分别为 $250 \sim 1750$ 及 $250 \sim 1600 \mathrm{~K}$. 在 $250 \sim 1000 \mathrm{~K}$ 的 温度范围内, 反应 I (1)与其竞争反应 II (1)的反应速率及平衡转化率差别很大, 具有很好 的选择性, 更有利于二溴卡宾插入乙醛 $\mathrm{C}_{\alpha}-\mathrm{H}$ 键生成产物 $\mathrm{P} 1$ 的反应 I (1)的发生, 而在 $1000 \sim 1600 \mathrm{~K}$ 范围内，反应没有明显的选择优势.
关键词

二溴卡宾

乙醛

插入反应

密度泛函理论

热力学与动力学性质
卡宾化学因其在有机理论上的重要性以及在药物 和张力小环等复杂化合物合成上的广泛应用, 多年来 一直备受人们的广泛关注 ${ }^{[1-4]}$. 目前, 人们已对卡宾与 碳碳双键、碳氮双键等多重键的反应及卡宾的环加成反 应进行了系统的研究 ${ }^{[57]}$. 有关卡宾与 $\mathrm{O}-\mathrm{H}$ 键的插入 反应研究也在逐步深入 ${ }^{[8,9]}$, 例如, Fang等人 ${ }^{[9]}$ 运用量子 化学方法对亚烷基卡宾与甲醇 $\mathrm{O}-\mathrm{H}$ 键的插入反应进行 了研究. 卡宾与 $\mathrm{C}-\mathrm{H}$ 键的插入反应是现代有机化学反 应中最令人感兴趣的反应之一. 人们已对卡宾与烃类 及其他有机物中 $\mathrm{C}-\mathrm{H}$ 键的插入反应进行了研究 ${ }^{[3,10,11]}$, 林启君等人 ${ }^{[12 \sim 14]}$ 对卡宾与醚类有机化合物 $\mathrm{C}-\mathrm{H}$ 键的插 入反应也进行了系统的理论研究. 量子化学计算方法 是研究化学反应机理的一种有效手段 ${ }^{[15 \sim 17]}$, 人们采用 量子化学计算方法, 利用经Wigner校正的Eyring过渡态 理论和统计热力学方法研究反应的热力学与动力学性 质 ${ }^{[15,18]}$, 并取得了一些有意义的结果. 在有关卡宾与碳 氢键插入反应的研究中, 简单醛类是很好的研究对象,
有关乙醛与 $\mathrm{C}-\mathrm{H}$ 键的插入已有报道 ${ }^{[19]}$, 但迄今为止, 人们对卡宾与醛类化合物 $\mathrm{C}-\mathrm{H}$ 键插入反应的实验和理 论研究甚少. 前文 ${ }^{[20]}$ 用量子化学计算方法系统研究了 单重态二溴卡宾与甲醛 $\mathrm{C}-\mathrm{H}$ 键的插入反应. 为了进一 步探求卡宾与醛类化合物碳氢键的插入反应规律, 并 理解其反应的热力学与动力学性质, 本文在 B3LYP/6-31G(d)水平上对单重态 $\mathrm{CH}_{2}$ 及单、三重态 $\mathrm{CBr}_{2}$ 与 $\mathrm{CH}_{3} \mathrm{CHO}$ 的插入反应机理进行了探讨, 研究了二溴 卡宾与乙醛反应的热力学与动力学性质, 以使人们对 卡宾与醛类化合物 $\mathrm{C}-\mathrm{H}$ 键的插入反应过程有一个系统 而深刻的认识.

\section{1 计算方法}

利用Gaussian 2003 程序, 考虑到密度泛函理论 (DFT)在几何构型优化和过渡态计算中的可靠性 ${ }^{[21]}$, 我们在B3LYP/6-31G(d)水平上对反应势能面上各驻 点的构型进行了全参数优化, 在 $6-31 \mathrm{G}(\mathrm{d})$ 及 $6-31 \mathrm{G}(\mathrm{d}$, 
p)基组水平上计算了反应势能面上各物种的 $\operatorname{CCSD}(\mathrm{T})$ 单点能量, 同时用内禀反应坐标(IRC)和频率分析方 法对过渡态进行了确认. 另外, 在MP $2 / 6-31 \mathrm{G}(\mathrm{d})$ 水平 下优化的主反应通道各物种的构型参数与 B $3 \mathrm{LYP} /$ 6-31G(d)水平下的一致, 进一步说明主反应通道中各 型体IM1.1，TS1.1，P1，IM4.1，TS4.1 及 P4 真实存在. 本文根据统计热力学方法和用Wigner校正的Eyring 过渡态理论, 利用自编程序, 计算反应的热力学函数 的变化和反应速率常数 $k(T)$.

\section{2 结果与讨论}

考虑了卡宾与乙醛中 $\alpha-\mathrm{C}$ 的 $\mathrm{C}-\mathrm{H}$ 键 $\left(\mathrm{C}_{\alpha}-\mathrm{H}\right)$ 及 $\beta-\mathrm{C}$ 的 $\mathrm{C}-\mathrm{H}$ 键 $\left(\mathrm{C}_{\beta}-\mathrm{H}\right)$ 的两种插入方式, 为了叙述方 便, 把卡宾 $\mathrm{C}$ 记为 $\mathrm{C}^{*}$. 同时, 我们考虑了二溴卡宾与 乙醛插入反应的单重态及三重态反应势能面, 其中 三重态势能面不重要, 所以主要介绍单重态势能面.

\section{1 单重态势能面}

(1) 二溴卡宾与乙醛的插入反应. 二溴卡宾插 入反应中部分反应物种的优化构型及势能剖面分别 如图 1 和 2 所示. 相应构型的能量及过渡态的唯一虚 频率列于表 1 , 图 1 中斜体参数为 MP2/6-31G(d)方法 的计算结果.

反应 I: 单重态二溴卡宾与乙醛 $\mathrm{C}_{\alpha}-\mathrm{H}$ 键的插 入反应.

二溴卡宾与乙醛 $\mathrm{C}_{\alpha}-\mathrm{H}$ 键插入生成 $\mathrm{P} 1$ 的反应 [反应 I (1)] 路径为 Path 1. 由图 1 和 2 可以看出, $\mathrm{CBr}_{2}$ 与 $\mathrm{CH}_{3} \mathrm{CHO}$ 接近, 先形成前期复合物 IM1.1, 其中 $\mathrm{C}^{*}$ 与 $\mathrm{CH}_{3} \mathrm{CHO}$ 中将被插入的 $\mathrm{C}_{\alpha}-\mathrm{H}$ 键上的碳原子 $\mathrm{C} 2$ 相距 $0.3239 \mathrm{~nm}, \mathrm{C}_{\alpha}-\mathrm{H}$ 键键长为 $0.1120 \mathrm{~nm}$, 这是一 不稳定的中间复合物, 能量比分离的 $\mathrm{CBr}_{2}$ 与 $\mathrm{CH}_{3} \mathrm{CHO}$ 能量之和低 $13.2 \mathrm{~kJ} \cdot \mathrm{mol}^{-1}, \mathrm{IM} 1.1$ 中 $\mathrm{C}^{*}-\mathrm{C} 2$ 键的键电荷值仅为 0.01 , 二溴卡宾与乙醛只有很弱的 相互作用. 随着 $\mathrm{CBr}_{2}$ 与 $\mathrm{CH}_{3} \mathrm{CHO}$ 的进一步靠近, 形 成反应的过渡态 TS1.1. 在 TS1.1 构型中, $\mathrm{C}^{*}-\mathrm{C} 2$ 间 距为 $0.2474 \mathrm{~nm}$, 被插入的 $\mathrm{C}_{\alpha}-\mathrm{H}$ 键拉长为 $0.1604 \mathrm{~nm}$, 键电荷值为 0.08 , 而 $\mathrm{H} 10$ 与 $\mathrm{C} *$ 则相距 $0.1160 \mathrm{~nm}$, 键 电荷值为 0.26 , 这说明过渡态与产物 $\mathrm{P} 1$ 态构型较为 相似. TS1.1 有唯一的虚频率 690i, 根据 TS1.1 的内禀 反应坐标(IRC)的计算结果和所得初始平衡构型的进 一步优化可知, TS1.1 连接着 IM1.1 和 P1. 该途径主 要是 2, 4- $\mathrm{H}$ 的转移和 $\mathrm{C}^{*}-\mathrm{C} 2$ 键的缩短成键过程.

从势能面图 2 上看, $\mathrm{P} 1$ 异构化为 $\mathrm{P} 2$ 和 $\mathrm{P} 3$ 的反
应 $[反$ 应 I (2)]途径均有较高的势垒, 且速控步骤过渡 态与反应物的能量基本相等, 所以 $\mathrm{P} 1$ 异构化为 $\mathrm{P} 2$ 和 P3 的途径 Path2 和 Path3 将难以发生.

综上所述, 单重态 $\mathrm{CBr}_{2}$ 与乙醛 $\mathrm{C}_{\alpha}-\mathrm{H}$ 键插入反 应的主要通道为生成 $\mathrm{P} 1$ 的反应 I (1). Kollmar和 Jeziorek等人 ${ }^{[22,23]}$ 设想卡宾与烃中碳氢键的插入反应 机理可分成两步，第一步为亲电反应，第二步为亲核 反应. 林启君等人 ${ }^{[12 ~ 14]}$ 对卡宾与醚类 $\mathrm{C}-\mathrm{H}$ 键的插入 进行研究后指出, 其插入反应为一亲电-亲核过程. 在反应 I (1)中，体系分子片 $\mathrm{CBr}_{2}$ 中 3 个原子占有的 $\mathrm{NPA}^{[24,25]}$ 电荷之和由起始的 0.000 变为 -0.048 , 再由 -0.048 变到 -0.404 , 最后由 -0.404 变到 -0.351 , 这说 明反应 I (1)具有亲电-亲核的反应特征. 从优化结构 图 1 可以看出, 从反应物 $\mathrm{R}$ 开始, 历经 $\mathrm{IM} 1.1 \rightarrow \mathrm{TS} 1.1$ $\rightarrow \mathrm{P} 1$, 卡宾 $\mathrm{C}^{*}$ 原子完成了 $\mathrm{sp}^{2}$ 到 $\mathrm{sp}^{3}$ 杂化的转变.

反应 II : 单重态二溴卡宾与乙醛 $\mathrm{C}_{\beta}-\mathrm{H}$ 键的插 入反应.

二溴卡宾与乙醛 $\mathrm{C}_{\beta}-\mathrm{H}$ 键插入生成 $\mathrm{P} 4$ 的反 应[反应 II (1)] 路径为 Path 4. 由图 1 和 2 可以看出, $\mathrm{CBr}_{2}$ 与 $\mathrm{CH}_{3} \mathrm{CHO}$ 接近, 形成前期复合物 IM4.1, 其中 $\mathrm{C}^{*}$ 与 $\mathrm{CH}_{3} \mathrm{CHO}$ 中将被插入的 $\mathrm{C}_{\beta}-\mathrm{H}$ 键上的碳原子 $\mathrm{C} 1$ 相距 $0.4433 \mathrm{~nm}, \mathrm{C}_{\beta}-\mathrm{H}$ 键键长为 $0.1098 \mathrm{~nm}$, 这也是 一不稳定的中间复合物, 能量比分离的 $\mathrm{CBr}_{2}$ 与 $\mathrm{CH}_{3} \mathrm{CHO}$ 能量之和低 $13.8 \mathrm{~kJ} \cdot \mathrm{mol}^{-1}, \mathrm{IM} 4.1$ 中 $\mathrm{C}^{*}-\mathrm{C} 1$ 键的键电荷值为 0.00 , 和 IM1.1 相比, 反应二溴卡宾 与乙醛的相互作用更弱. 随着 $\mathrm{CBr}_{2}$ 与 $\mathrm{CH}_{3} \mathrm{CHO}$ 的进 一步靠近, 形成过渡态 TS4.1. 在过渡态 TS4.1 构型 中, $C^{*}-\mathrm{C} 1$ 间距缩短为 $0.2447 \mathrm{~nm}$, 键电荷值为 0.06 , 被插入的 $\mathrm{C}_{\beta}-\mathrm{H}$ 键拉长为 $0.1497 \mathrm{~nm}$, 而 $\mathrm{H} 7$ 与 $\mathrm{C}$ *则 相距 $0.1190 \mathrm{~nm}$, 键电荷值为 0.26 , 这说明过渡态与 产物 P4态构型较为相似. TS4.1 有唯一的虚频率 (902i), 根据 TS4.1 的内禀反应坐标(IRC)计算结果和 所得初始平衡构型的进一步优化可知, TS4.1 连接着 IM4.1 和 P4. 该途径主要是 $1,4-\mathrm{H}$ 的转移和 $\mathrm{C} *-\mathrm{C} 1$ 键的缩短成键过程.

从图 2 可以看出, P4 异构化分解为 P5 和 P6 的反 应[反应 II (2)]途径均有较高的势垒, 且速控步骤过渡 态与反应物的能量相当, 所以 P4 异构化分解为 P5 和 P6 的途径 Path 5 和 Path 6 的可能性减小.

综上所述, 单重态 $\mathrm{CBr}_{2}$ 与 $\mathrm{CH}_{3} \mathrm{CHO}$ 中 $\mathrm{C}_{\beta}-\mathrm{H}$ 键 插入的主要通道为生成 P4 的反应 II (1). 在反应 II (1) 中, 体系分子片 $\mathrm{CBr}_{2}$ 中 3 个原子占有的 NPA 电荷之 


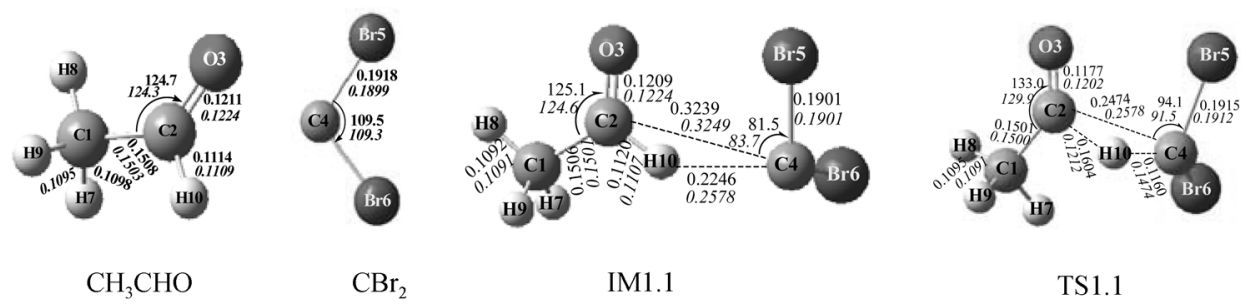

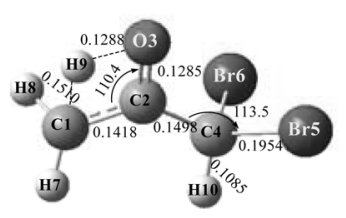

TS23.1

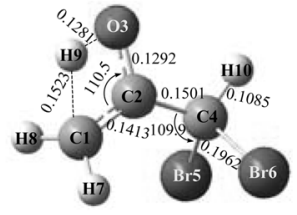

TS3.1

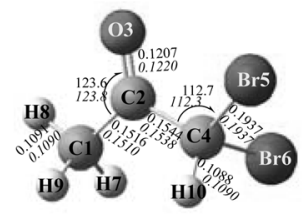

P1

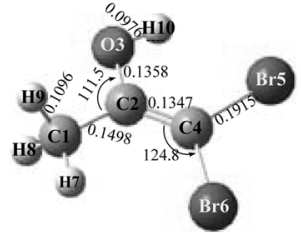

P2

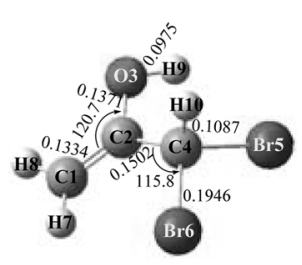

P3

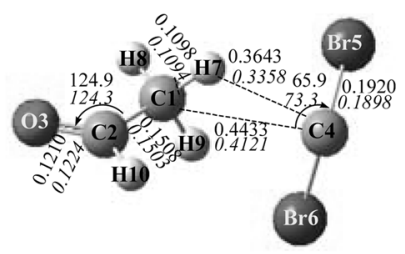

IM4. 1

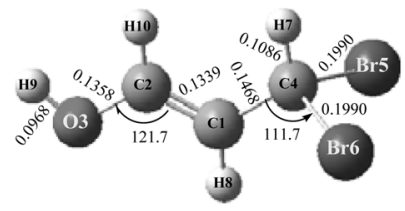

IM6.3

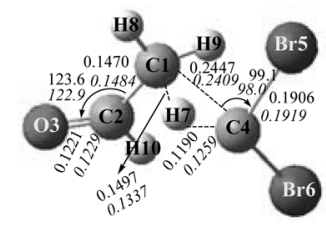

TS4. 1

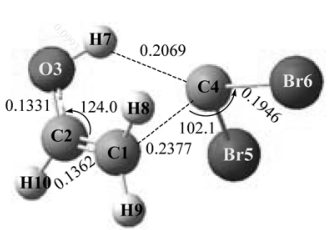

TS5.1

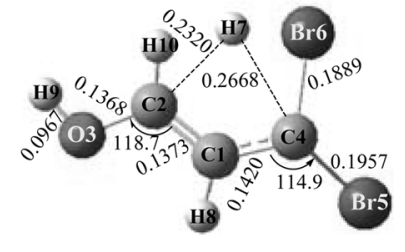

TS6.4

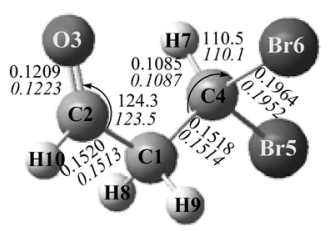

P4

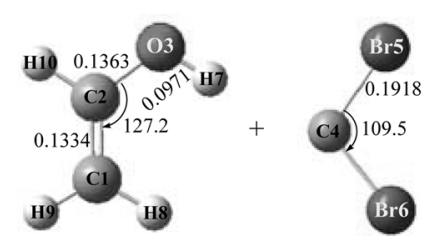

P5

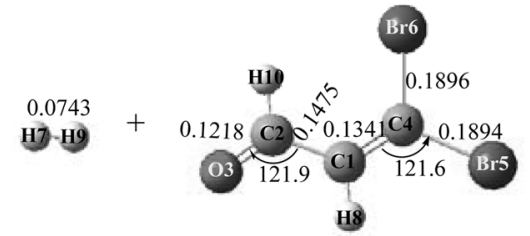

P6

图 1 单重态 $\mathrm{CBr}_{2}$ 与 $\mathrm{CH}_{3} \mathrm{CHO}$ 插入反应中部分物种的构型示意图

键长: $\mathrm{nm}$, 键角: $\left(^{\circ}\right)$

和由反应物 $\mathrm{CBr}_{2}$ 中的 0.000 变为 IM4 4 中的 0.007 , 该 过程可认为是亲核过程; 随即, 分子片 $\mathrm{CBr}_{2}$ 的电荷 之和由 IM4.1 中的 0.007 变到 TS4.1 中的 -0.165 , 最 后由 -0.165 变为产物 P4中的 -0.308 , 该过程可认为 是一亲电过程, 这说明反应 II (1)具有亲核-亲电的反 应特征. 在此过程中, 从 $\mathrm{R} \rightarrow \mathrm{IM} 4.1$, 分子片 $\mathrm{CBr}_{2}$ 流出 的电荷布居数为 0.007 , 而在接受电荷的乙醛分子片 中, 除被插入的 $\mathrm{C}_{\beta}-\mathrm{H}$ 键中的 $\mathrm{H} 7$ 原子的电荷布居由
反应物乙醛分子中的 0.252 变为了 0.247 外, 其他原 子的电荷布居基本未变, 说明该亲核过程主要是 $\mathrm{CBr}_{2}$ 分子片中的电子流向了乙醛分子片中的 $\mathrm{H} 7$ 原子; 从 IM4.1 开始, 历经 TS4.1 到生成产物 P4, 与卡宾平 面垂直的空的卡宾 $\mathrm{p}$ 轨道从指向被插入的 $\mathrm{C}_{\beta}-\mathrm{H}$ 键 偏 $\mathrm{H} 4$ 原子逐渐转到基本正指向 $\mathrm{C} 1$ 原子, 该过程有 利于 IM4.1 中高负电荷布居的 C1(-0.783) 将电子逐渐 流入低正电荷布居的分子片 $\mathrm{CBr}_{2}(0.07)$ 的空 $\mathrm{p}$ 轨道, 


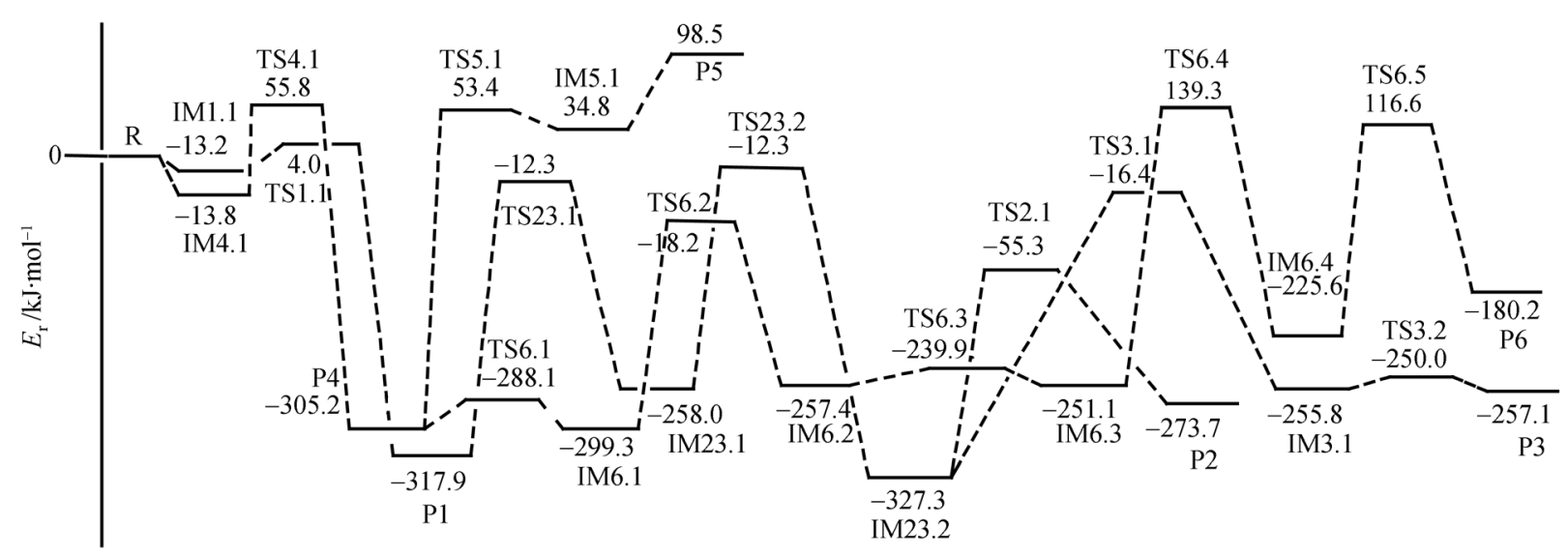

图 2 单重态 $\mathrm{CBr}_{2}$ 与 $\mathrm{CH}_{3} \mathrm{CHO}$ 插入反应的能量变化示意图

$\operatorname{CCSD}(\mathrm{T}) / 6-31 \mathrm{G}(\mathrm{d}, \mathrm{p})$ 能量

表 1 单重态 $\mathrm{CBr}_{2}$ 与 $\mathrm{CH}_{3} \mathrm{CHO}$ 插入反应中部分物种的总能量 $\left(E_{\mathrm{t}}(\mathrm{a} . \mathrm{u}).\right)$ 和相对能量 $\left(E_{\mathrm{r}} / \mathrm{kJ} \cdot \mathrm{mol}^{-1}\right)$ 及过渡态势垒 $\left(E^{\ddagger} / \mathbf{k J} \cdot \mathbf{m o l}^{-1}\right)^{\mathbf{a})}$

\begin{tabular}{|c|c|c|c|c|c|c|c|c|}
\hline \multirow{2}{*}{ 物种 } & \multicolumn{2}{|c|}{ B3LYP/6-31G(d) } & \multicolumn{2}{|c|}{$\operatorname{CCSD}(\mathrm{T}) / 6-31(\mathrm{~d})$} & \multicolumn{3}{|c|}{$\operatorname{CCSD}(T) / 6-31(d, p)$} & \multirow{2}{*}{$v / \mathrm{cm}^{-1}$} \\
\hline & $E_{\mathrm{t}}$ & $E_{\mathrm{r}}$ & $E_{\mathrm{t}}$ & $E_{\mathrm{r}}$ & $E_{\mathrm{t}}$ & $E_{\mathrm{r}}$ & $E^{\neq}$ & \\
\hline $\mathrm{R} 1+\mathrm{R} 2$ & -5335.17164 & 0.0 & -5331.23497 & 0.0 & -5331.26807 & 0.0 & & \\
\hline IM1.1 & -5335.17638 & -12.4 & -5331.23987 & -12.9 & -5331.27310 & -13.2 & & \\
\hline IM4.1 & -5335.17461 & -7.8 & -5331.23999 & -13.2 & -5331.27334 & -13.8 & & \\
\hline IM6.3 & -5335.25948 & -230.6 & -5331.32671 & -240.9 & -5331.36371 & -251.1 & & \\
\hline $\mathrm{TS} 1.1$ & -5335.16727 & 11.5 & -5331.23090 & 10.7 & -5331.26653 & 4.1 & 17.26 & $690 \mathrm{i}$ \\
\hline TS23.1 & -5335.17371 & -5.4 & -5331.23625 & -3.4 & -5331.23622 & -12.3 & 305.53 & $2249 \mathrm{i}$ \\
\hline TS3.1 & -5335.17486 & -8.5 & -5331.23780 & -7.4 & -5331.27433 & -16.4 & 310.85 & $2247 \mathrm{i}$ \\
\hline TS4.1 & -5335.14868 & 60.3 & -5331.21099 & 63.0 & -5331.24682 & 55.8 & 69.63 & $902 \mathrm{i}$ \\
\hline TS5.1 & -5335.15231 & 50.8 & -5331.21080 & 63.6 & -5331.24775 & 53.4 & 358.56 & $111 \mathrm{i}$ \\
\hline TS6.4 & -5335.11974 & 136.3 & -5331.17895 & 147.1 & -5331.21503 & 139.3 & 390.36 & $665 \mathrm{i}$ \\
\hline P1 & -5335.28543 & -298.8 & -5331.35605 & -317.9 & -5331.38914 & -317.9 & & \\
\hline P2 & -5335.26802 & -253.0 & -5331.33550 & -263.9 & -5331.37231 & -273.7 & & \\
\hline P3 & -5335.25980 & -231.5 & -5331.32892 & -246.7 & -5331.36599 & -257.1 & & \\
\hline P4 & -5335.27906 & -282.0 & -5331.35135 & -305.6 & -5331.38432 & -305.2 & & \\
\hline P5 & -5335.14628 & 66.6 & -5331.23405 & 2.4 & -5331.23053 & 98.5 & & \\
\hline P6 & -5335.17164 & -168.2 & -5331.30649 & -187.8 & -5331.33671 & -180.2 & & \\
\hline
\end{tabular}

a) $E_{\mathrm{t}}=E_{\text {species }}+\mathrm{ZPE}, E_{\mathrm{r}}=E_{\mathrm{t}}-E(\mathrm{R} 1+\mathrm{R} 2)$

至产物 $\mathrm{P} 4$ 中, $\mathrm{C} 1$ 和分子片 $\mathrm{CBr}_{2}$ 的电荷布居分别为 -0.602 和 -0.308 , 完成了亲电过程. 从优化构型图 1 可以看出, 从反应物 $\mathrm{R}$ 开始, 历经 $\mathrm{IM} 4.1 \rightarrow \mathrm{TS} 4.1 \rightarrow \mathrm{P} 4$ 过程, 卡宾碳原子 $\mathrm{C} *$ 完成了从 $\mathrm{sp}^{2}$ 到 $\mathrm{sp}^{3}$ 杂化的转变.

从以上讨论可知, 单重态二溴卡宾与乙醛插入 反应有两条通道, 一是卡宾碳原子 $\mathrm{C} *$ 与乙醛 $\mathrm{C}_{\alpha}-\mathrm{H}$ 键插入发生反应 I (1)生成 P1 的通道 Path 1 , 另一是 C*插入乙醛 $\mathrm{C}_{\beta}-\mathrm{H}$ 键发生反应 II (1)生成 P4 的通道 Path 4. Path 1 和 Path 4 均为一过渡态一中间体过程, 这与二溴卡宾和甲醛的插入反应一致. 从此两个反 应的势垒高低可以清楚地看出, $\mathrm{C}^{*}$ 与乙醛 $\mathrm{C}_{\alpha}-\mathrm{H}$ 键
插入生成 P1 的反应更容易发生. 从前面讨论可知, 卡宾 $C^{*}$ 与乙醛 $C_{\alpha}-H$ 键及 $C_{\beta}-H$ 键的插入分别具有 亲电-亲核与亲核-亲电的特点, 在反应 I (1)中, $\mathrm{C} 2$ 原 子的高正电荷布居(IM1.1：0.427；TS1.1：0.577；P1： 0.552 ) 导致其亲核反应容易发生，而在反应 II (1)中, 从 IM4.1 开始, 虽然卡宾空的 $\mathrm{p}$ 轨道逐渐正指向高负 电子布居的 $\mathrm{C} 1$ 原子, 有利于 $\mathrm{C} 1$ 原子中的电子向空 $\mathrm{p}$ 轨道的流入, 但毕竟在此过程中 $\mathrm{CBr}_{2}$ 分子片的电荷 布居一直为负, 对电子从 $\mathrm{C} 1$ 到空 $\mathrm{p}$ 轨道的流入有一 定的延滞作用, 故 $\mathrm{CBr}_{2}$ 与 $\mathrm{C}_{\beta}-\mathrm{H}$ 键插入的反应 I (1) 势垒高并不偶然, 这也是对前文反应机理分析的进 
一步证实.

(2) 卡宾与乙醛的插入反应. 卡宾插入反应中 部分反应物种的优化构型及势能剖面分别如图 3 和 4 所示. 从图可以看出, 与反应 I (1)相似, 首先经一无 能垒过程, 卡宾与乙醛形成前期复合物 IM1，IM1 再 经过一 $17.3 \mathrm{~kJ} \cdot \mathrm{mol}^{-1}$ 的小势垒过渡态 $\mathrm{TS} 1$, 生成中间 体 IM2，此过程放热 $477.2 \mathrm{~kJ} \cdot \mathrm{mol}^{-1}$. IM2 可通过 TS2 进一步异构化为与 $\mathrm{P} 4$ 具有相似构型的产物 ${ }^{\mathrm{H}} \mathrm{P} 1$, 此 过程主要是二面角 $\mathrm{D} 4123$ 的变化过程. ${ }^{\mathrm{H}} \mathrm{P} 1$ 再经过 4,3-H 的转移和 $\angle 412$ 的减小, 生成产物 ${ }^{\mathrm{H}} \mathrm{P} 2$. 可以看 出, 虽然 TS3 较反应物的能量低 $135.7 \mathrm{~kJ} \cdot \mathrm{mol}^{-1}$, 但
该过程的势垒较高 $\left(347.1 \mathrm{~kJ} \cdot \mathrm{mol}^{-1}\right)$, 所以 ${ }^{\mathrm{H}} \mathrm{P} 1$ 异构化 为产物 ${ }^{\mathrm{H}} \mathrm{P} 2$ 的可能性减小, 但产物中 ${ }^{\mathrm{H}} \mathrm{P} 2$ 也有一定的 比例.

图 4 表明, 卡宾与乙醛中 $\mathrm{C}-\mathrm{H}$ 的插入也存在生 成产物 ${ }^{\mathrm{H}} \mathrm{P} 3$ (与 $\mathrm{P} 1$ 构型相似)和 ${ }^{\mathrm{H}} \mathrm{P} 4$ (与 $\mathrm{P} 6$ 构型相似) 的反应通道, ${ }^{\mathrm{H}} \mathrm{P} 3$ 可进一步异构化为 ${ }^{\mathrm{H}} \mathrm{P} 5$ 和 ${ }^{\mathrm{H}} \mathrm{P} 6$ (与 $\mathrm{P} 3$ 构型相似), 但这些反应通道中速控步骤的能垒很高 $\left(400 \mathrm{~kJ} \cdot \mathrm{mol}^{-1}\right.$ 以上), 且速控步骤中过渡态的能量与 反应物的相当, 故卡宾与乙醛插入生成 ${ }^{\mathrm{H}} \mathrm{P} 3 \sim 6$ 的程度 减小, 丙醛 ${ }^{\mathrm{H}} \mathrm{P} 1$ 是卡宾与乙醛的插入反应的主反应产 物, 次要产物是 ${ }^{\mathrm{H}} \mathrm{P} 2$.

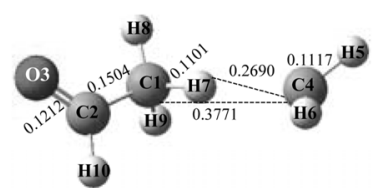

IM1

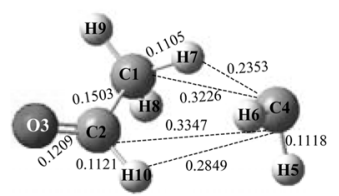

TS4

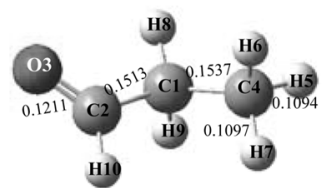

IM2

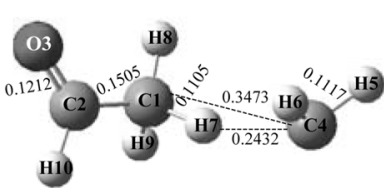

TS1

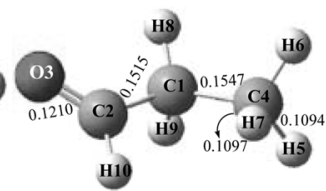

TS2

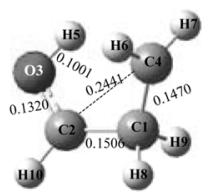

TS3

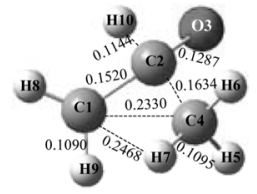

TS5

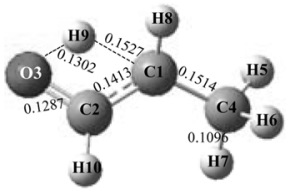

TS6

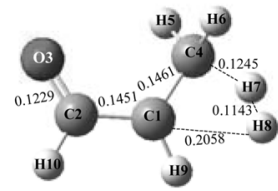

TS9

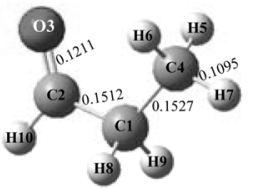

HP1
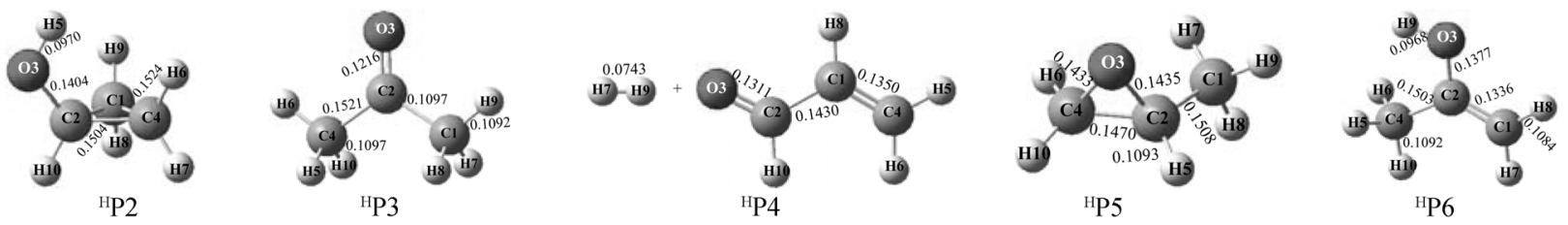

图 $3 \mathrm{CH}_{2}$ 与 $\mathrm{CH}_{3} \mathrm{CHO}$ 中 $\mathrm{C}-\mathrm{H}$ 插入反应部分物种的构型示意图(键长: $\mathrm{nm}$ )

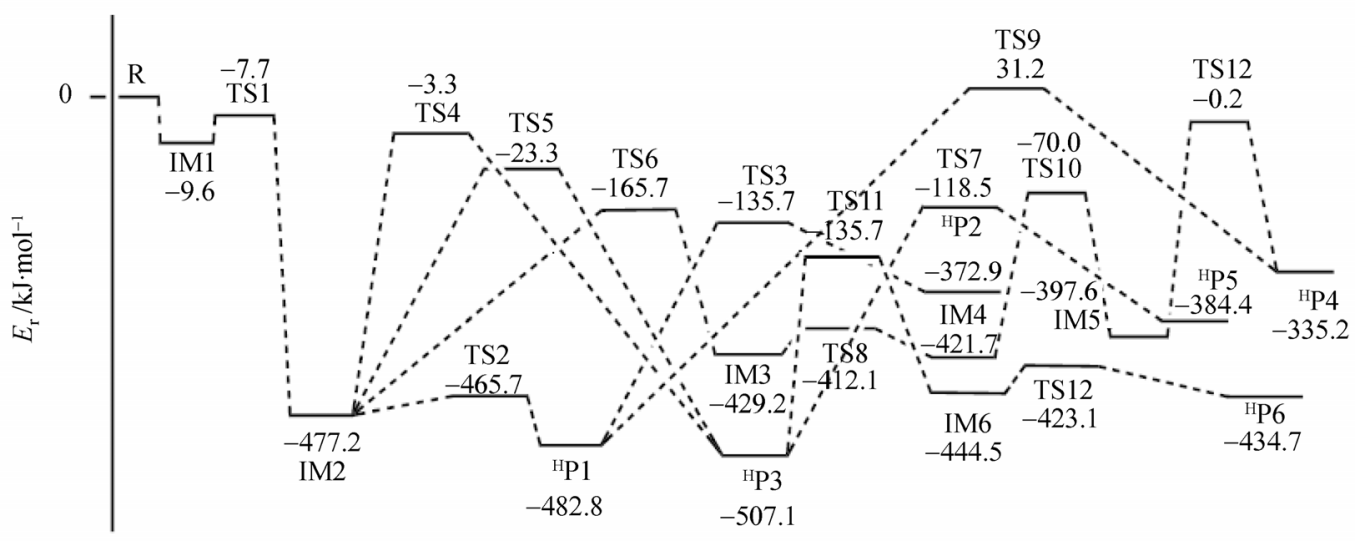

图 $4 \mathrm{CH}_{2}$ 与 $\mathrm{CH}_{3} \mathrm{CHO}$ 中 $\mathrm{C}-\mathrm{H}$ 键插入反应的能量变化示意图

$\operatorname{CCSD}(\mathrm{T}) / 6-31 \mathrm{G}(\mathrm{d}, \mathrm{p})$ 能量 
$\mathrm{NPA}$ 分析表明, 过程 $\mathrm{R} \rightarrow \mathrm{IM} 1 \rightarrow \mathrm{TS} 1 \rightarrow \mathrm{IM} 2$ 中体 系分子片 $\mathrm{CH}_{2}$ 的电荷之和的变化过程为 $0.00 \rightarrow 0.015$ $\rightarrow 0.013 \rightarrow-0.203$. 因此, 卡宾 $\mathrm{CH}_{2}$ 与乙醛 $\mathrm{CH}_{3} \mathrm{CHO}$ 的 插入反应具有亲核-亲电的反应特征. 从图 3 可以看 出, 随着 $\mathrm{C} 1$ 与 $\mathrm{C} 4$ 的相互靠近成键, 键长和键电荷值 变化分别为 $\operatorname{IM} 1(0.3771 \mathrm{~nm}) \rightarrow \operatorname{TS} 1(0.3473 \mathrm{~nm}) \rightarrow$ $\mathrm{IM} 2(0.1537 \mathrm{~nm})$ 和 $\mathrm{IM} 1(0.01) \rightarrow \mathrm{TS} 1(0.11) \rightarrow \operatorname{IM} 2(0.34)$. 从反应物 $\mathrm{R}$ 开始至中间体 IM2, 卡宾碳原子完成了从 $\mathrm{sp}^{2}$ 到 $\mathrm{sp}^{3}$ 杂化的转变.

\section{2 三重态势能面}

三重态二溴卡宾与乙醛 $\mathrm{C}_{\alpha}-\mathrm{H}$ 插入反应中部分 反应物种的优化构型及势能剖面分别如图 5 和 6 所示.

从图 5 和 6 可以看出, 三重态 $\mathrm{CBr}_{2}$ 与 $\mathrm{CH}_{3} \mathrm{CHO}$ 首先形成一中间体 ${ }^{3} \mathrm{IM} 1$, 释能 $17.4 \mathrm{~kJ} \cdot \mathrm{mol}^{-1},{ }^{3} \mathrm{IM} 1$ 越 过一 $26.4 \mathrm{~kJ} \cdot \mathrm{mol}^{-1}$ 的势垒, 生成产物 ${ }^{3} \mathrm{P} 1,{ }^{3} \mathrm{P} 1$ 可进一 步或异构化、或异构化分解为产物 ${ }^{3} \mathrm{P} 2,{ }^{3} \mathrm{P} 3$ 及 ${ }^{3} \mathrm{P} 4$. 由 于 ${ }^{3} \mathrm{P} 1$ 进一步反应分别经历过渡态 ${ }^{3} \mathrm{TS} 2,{ }^{3} \mathrm{TS} 3$ 及 ${ }^{3} \mathrm{TS} 4$
时, 需翻越的势垒较高, 且过渡态能量均高于反应物 $100 \mathrm{~kJ} \cdot \mathrm{mol}^{-1}$ 以上, 对后续反应的进行没有贡献, 故 ${ }^{3} \mathrm{P} 1$ 后续反应进行的可能性减小. 相比之下, ${ }^{3} \mathrm{P} 1$ 发生 逆反应的势垒较小 $\left(139.6 \mathrm{~kJ} \cdot \mathrm{mol}^{-1}\right)$, 一旦有 ${ }^{3} \mathrm{P} 1$ 生成, ${ }^{3} \mathrm{P} 1$ 又较快地经过逆反应过程分解为反应物 $\mathrm{R}$. 因此, 相对于单重态势能面来说, 三重态 $\mathrm{CBr}_{2}$ 与 $\mathrm{CH}_{3} \mathrm{CHO}$ 中 $\mathrm{C}_{\alpha}-\mathrm{H}$ 键的插入对整个反应的贡献不大. 我们没 有得到三重态 $\mathrm{CBr}_{2}$ 与 $\mathrm{CH}_{3} \mathrm{CHO}$ 中 $\mathrm{C}_{\beta}-\mathrm{H}$ 插入反应的 势能面, 这可能是因为和单重态 $\mathrm{CBr}_{2}$ 相比, 三重态 $\mathrm{CBr}_{2}$ 中 $\mathrm{C}$ 原子的较高负电子布居 $(-0.2973)$ 与荷负电 的乙醛 $\mathrm{C} 1$ 原子之间具有更强的静电斥力的缘故. 因 此, 我们认为三重态二溴卡宾与乙醛 $\mathrm{C}-\mathrm{H}$ 的插入反 应不重要, 以下热力学与动力学性质的讨论只针对 单重态二溴卡宾与乙醛 $\mathrm{C}-\mathrm{H}$ 的插入反应.

\section{3 反应 I (1)及 II (1)的热力学与动力学性质}

由图 2 和 4 可知, 生成产物 P1 的反应 I (1)及 P4 的反应 II (1)的速控步骤分别为 $\mathrm{IM} 1.1 \rightarrow \mathrm{TS} 1.1 \rightarrow \mathrm{P} 1$

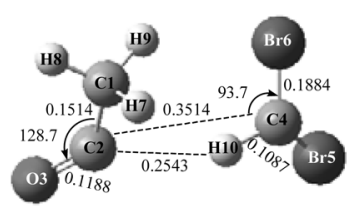

${ }^{3}$ IM1

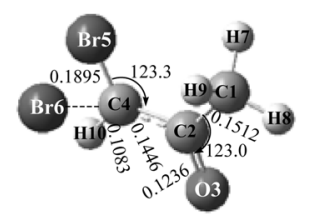

${ }^{3} \mathrm{P} 1$

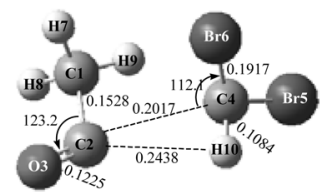

${ }^{3} \mathrm{TS} 1$

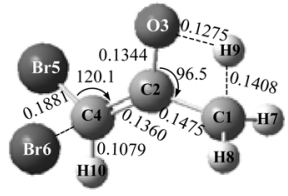

${ }^{3} \mathrm{TS} 2$

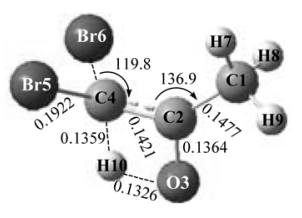

${ }^{3} \mathrm{TS} 3$

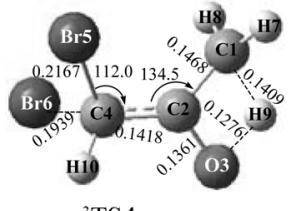

${ }^{3} \mathrm{TS} 4$
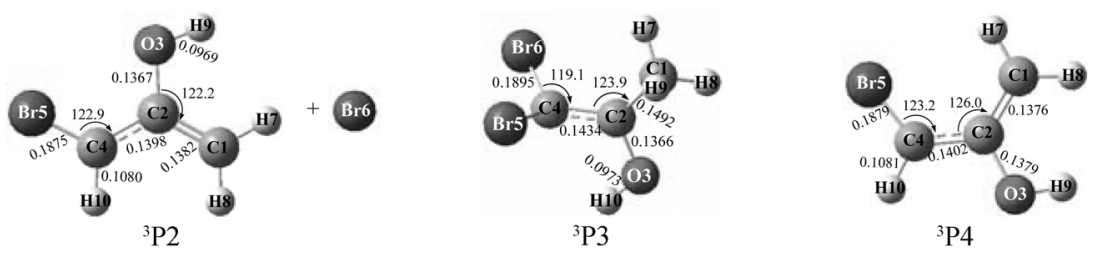

${ }^{3} \mathrm{P} 4$

图 5 三重态 $\mathrm{CBr}_{2}$ 与 $\mathrm{CH}_{3} \mathrm{CHO}$ 中 $\mathrm{C}_{\alpha}-\mathrm{H}$ 插入反应部分物种的构型示意图

键长: $\mathrm{nm}$, 键角: $\left(^{\circ}\right)$

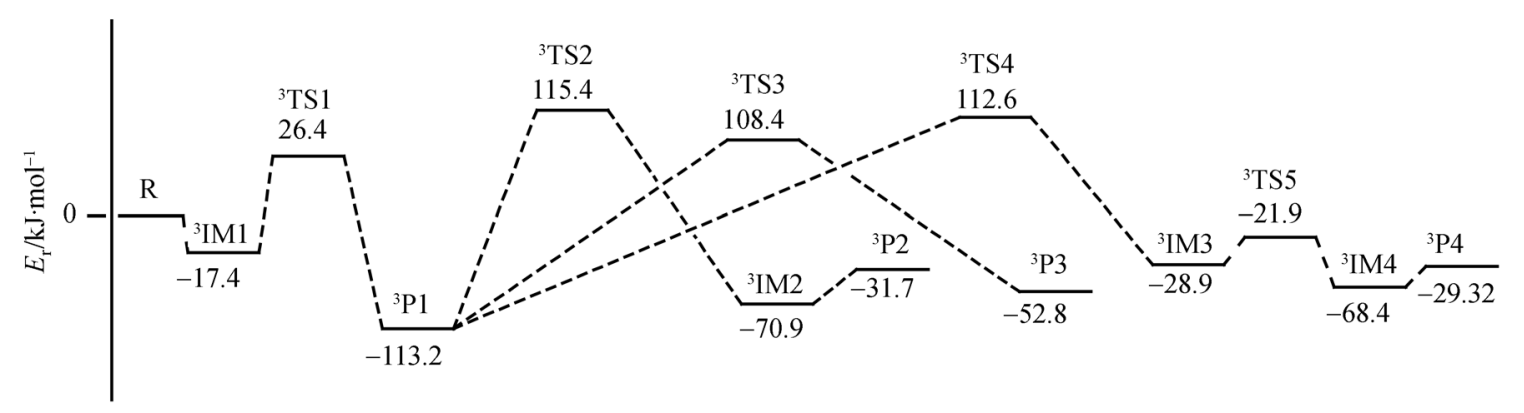

图 6 三重态 $\mathrm{CBr}_{2}$ 与 $\mathrm{CH}_{3} \mathrm{CHO}$ 中 $\mathrm{C}_{\alpha}-\mathrm{H}$ 键插入反应的能量变化示意图

$\operatorname{CCSD}(\mathrm{T}) / 6-31 \mathrm{G}(\mathrm{d}, \mathrm{p})$ 能量 
及 $\mathrm{IM} 4.1 \rightarrow \mathrm{TS} 4.1 \rightarrow \mathrm{P} 4$, 在 $1 \mathrm{~atm}(101325 \mathrm{~Pa})$ 和不同温 度下, 根据统计热力学方法和用 Wigner 校正的 Eyring 过渡态理论计算得到的反应 I (1)及 II (1)的热 力学函数改变值、体系标准摩尔活化摘 $\Delta_{\mathrm{r}}^{\neq} G_{\mathrm{m}}^{\ominus}$ 、标准 摩尔活化焓 $\Delta_{\mathrm{r}}^{\neq} H_{\mathrm{m}}^{\ominus}$ 、频率因子 $A$ 及 Wigner 校正因子 $g$ 分别列于表 2 和 3 .

由表 2 和 3 可以看出, $101325 \mathrm{~Pa}$ 时, 在所研究的 温度范围内, 反应 I (1)及 II (1)均是一熵减少且放热的 反应, 其自发温度范围分别为 100 1750 和 100 1600 K.
在低温时, 反应 I (1)及 II (1)的 Wigner 因子 $g$ 较大, $g$ 的引入校正了 Eyring 过渡态理论中未考虑到钻穿效 应而导致计算结果偏低的缺陷, 而在所研究的温度 范围内, 频率因子 $A$ 基本保持不变, 表明反应 I (1)及 II (1)均具有典型的 Arrhenius 反应特征. 由于过渡态 TS1.1 和 TS4.1 分别比中间体 IM1.1 和 IM4.1 的构 型紧凑, 所以反应 I (1)和 II (1)相应的 $\Delta_{\mathrm{r}}^{\neq} S_{\mathrm{m}}^{\ominus}$ 均为负值.

图 7(a)和(b)分别为反应 I (1)及 II (1)的平衡常数 $K$ 的对数值 $\log K$ 及速率常数 $k$ 的对数值 $\log k$ 随温度 $T$

表 2 单重态 $\mathrm{CBr}_{2}$ 与 $\mathrm{CH}_{3} \mathrm{CHO}$ 中 $\mathrm{C}_{\alpha}-\mathrm{H}$ 键插入反应 I (1)的热力学性质 ${ }^{\text {a) }}$

\begin{tabular}{|c|c|c|c|c|c|c|c|c|c|c|c|}
\hline \multirow{2}{*}{$T / \mathrm{K}$} & \multicolumn{3}{|c|}{$\mathrm{R} \rightarrow \mathrm{P} 1[$ I (1)-total] } & \multicolumn{8}{|c|}{$\mathrm{IM} 1.1 \rightarrow \mathrm{TS} 1.1 \rightarrow \mathrm{P} 1[\mathrm{I}(1)$-rate $]$} \\
\hline & $\Delta H^{\ominus}$ & $\Delta G^{\ominus}$ & $\Delta S^{\ominus}$ & $\Delta H^{\ominus}$ & $\Delta G^{\ominus}$ & $\Delta S^{\ominus}$ & $\Delta_{\mathrm{r}}^{\neq} H_{\mathrm{m}}^{\ominus}$ & $\Delta_{\mathrm{r}}^{\neq} S_{\mathrm{m}}^{\ominus}$ & $\Delta_{\mathrm{r}}^{\neq} G_{\mathrm{m}}^{\ominus}$ & $g$ & $A$ \\
\hline 100 & -300.930 & -284.407 & -165.223 & -287.897 & -284.145 & -37.518 & 22.561 & -33.003 & 25.861 & 1.0412 & $3.01 \times 10^{10}$ \\
\hline 250 & -301.625 & -259.061 & -170.258 & -290.611 & -277.024 & -54.348 & 21.038 & -42.890 & 31.761 & 1.0066 & $3.48 \times 10^{10}$ \\
\hline 400 & -301.098 & -233.612 & -168.715 & -292.441 & -268.337 & -60.262 & 20.193 & -45.572 & 38.422 & 1.0026 & $3.93 \times 10^{10}$ \\
\hline 550 & -299.822 & -208.501 & -166.037 & -293.604 & -259.087 & -62.759 & 19.431 & -47.192 & 45.387 & 1.0014 & $4.28 \times 10^{10}$ \\
\hline 700 & -298.136 & -183.801 & -163.336 & -294.389 & -249.580 & -64.013 & 18.628 & -48.482 & 52.565 & 1.0008 & $4.55 \times 10^{10}$ \\
\hline 850 & -296.225 & -159.491 & -160.863 & -294.957 & -239.926 & -64.742 & 17.775 & -49.585 & 59.922 & 1.0006 & $4.76 \times 10^{10}$ \\
\hline 1000 & -294.185 & -135.528 & -158.656 & -295.413 & -230.154 & -65.259 & 16.869 & -50.565 & 67.433 & 1.0004 & $4.92 \times 10^{10}$ \\
\hline 1150 & -292.058 & -111.883 & -156.674 & -295.799 & -220.350 & -65.608 & 15.916 & -51.453 & 75.087 & 1.0003 & $5.05 \times 10^{10}$ \\
\hline 1300 & -289.874 & -88.519 & -154.888 & -296.107 & -210.484 & -65.864 & 14.929 & -52.258 & 82.864 & 1.0002 & $5.14 \times 10^{10}$ \\
\hline 1450 & -287.645 & -65.406 & -153.268 & -296.390 & -200.601 & -66.061 & 13.902 & -53.006 & 90.761 & 1.0002 & $5.22 \times 10^{10}$ \\
\hline 1600 & -285.376 & -42.530 & -151.779 & -296.624 & -190.682 & -66.213 & 12.849 & -53.696 & 98.764 & 1.0002 & $5.29 \times 10^{10}$ \\
\hline 1750 & -283.079 & -19.870 & -150.405 & -296.855 & -180.729 & -66.358 & 11.770 & -54.342 & 106.869 & 1.0001 & $5.34 \times 10^{10}$ \\
\hline 1900 & -280.760 & 2.597 & -149.135 & -297.039 & -170.773 & -66.456 & 10.670 & -54.945 & 115.065 & 1.0001 & $5.38 \times 10^{10}$ \\
\hline
\end{tabular}

a) $\Delta H^{\ominus}: \mathrm{kJ} \cdot \mathrm{mol}^{-1}, \Delta G^{\ominus}: \mathrm{kJ} \cdot \mathrm{mol}^{-1}, \Delta S^{\ominus}: \mathrm{J} \cdot \mathrm{K}^{-1} \cdot \mathrm{mol}^{-1}, \Delta_{\mathrm{r}}^{\ddagger} H_{\mathrm{m}}^{\ominus}: \mathrm{kJ} \cdot \mathrm{mol}^{-1}, \Delta_{\mathrm{r}}^{\ddagger} S_{\mathrm{m}}^{\ominus}: \mathrm{J} \cdot \mathrm{K}^{-1} \cdot \mathrm{mol}^{-1}, \Delta_{\mathrm{r}}^{\neq} G_{\mathrm{m}}^{\ominus}: \mathrm{kJ} \cdot \mathrm{mol}^{-1}$

表 3 单重态 $\mathrm{CBr}_{2}$ 与 $\mathrm{CH}_{3} \mathrm{CHO}$ 中 $\mathrm{C}_{\beta}-\mathrm{H}$ 键插入反应 II (1)的热力学性质 a)

\begin{tabular}{|c|c|c|c|c|c|c|c|c|c|c|c|}
\hline \multirow{2}{*}{$T / \mathrm{K}$} & \multicolumn{3}{|c|}{$\mathrm{R} \rightarrow \mathrm{P} 4$ [ II (1)-total] } & \multicolumn{8}{|c|}{$\mathrm{IM} 4.1 \rightarrow \mathrm{TS} 4.1 \rightarrow \mathrm{P} 4[\mathrm{II}(1)$-rate $]$} \\
\hline & $\Delta H^{\ominus}$ & $\Delta G^{\ominus}$ & $\Delta S^{\ominus}$ & $\Delta H^{\ominus}$ & $\Delta G^{\ominus}$ & $\Delta S^{\ominus}$ & $\Delta_{\mathrm{r}}^{\neq} H_{\mathrm{m}}^{\ominus}$ & $\Delta_{\mathrm{r}}^{\neq} S_{\mathrm{m}}^{\ominus}$ & $\Delta_{\mathrm{r}}^{\neq} G_{\mathrm{m}}^{\ominus}$ & $g$ & $A$ \\
\hline 100 & -284.402 & -267.491 & -169.108 & -276.444 & -271.159 & -52.851 & 66.538 & -33.003 & 69.957 & 1.0703 & $4.21 \times 10^{10}$ \\
\hline 250 & -285.523 & -241.362 & -176.644 & -279.786 & -261.353 & -73.735 & 64.243 & -42.890 & 76.418 & 1.0112 & $3.03 \times 10^{10}$ \\
\hline 400 & -285.486 & -214.824 & -176.657 & -282.162 & -249.640 & -81.305 & 62.847 & -45.572 & 84.113 & 1.0044 & $3.48 \times 10^{10}$ \\
\hline 550 & -284.570 & -188.456 & -174.753 & -283.691 & -237.167 & -84.589 & 61.928 & -47.192 & 92.250 & 1.0023 & $3.93 \times 10^{10}$ \\
\hline 700 & -283.105 & -162.421 & -172.405 & -284.683 & -224.349 & -86.191 & 61.190 & -48.482 & 100.612 & 1.0014 & $4.28 \times 10^{10}$ \\
\hline 850 & -281.320 & -136.733 & -170.102 & -285.366 & -211.345 & -87.083 & 60.497 & -49.585 & 109.132 & 1.0010 & $4.55 \times 10^{10}$ \\
\hline 1000 & -279.343 & -111.382 & -167.961 & -285.859 & -198.241 & -87.618 & 59.788 & -50.565 & 117.772 & 1.0007 & $4.76 \times 10^{10}$ \\
\hline 1150 & -277.240 & -86.337 & -166.002 & -286.235 & -185.069 & -87.97 & 59.034 & -51.453 & 126.523 & 1.0005 & $4.92 \times 10^{10}$ \\
\hline 1300 & -275.055 & -61.573 & -164.217 & -286.529 & -171.855 & -88.211 & 58.234 & -52.258 & 135.376 & 1.0004 & $5.05 \times 10^{10}$ \\
\hline 1450 & -272.813 & -37.064 & -162.585 & -286.765 & -158.609 & -88.383 & 57.38 & -53.006 & 144.324 & 1.0003 & $5.14 \times 10^{10}$ \\
\hline 1600 & -270.521 & -12.791 & -161.081 & -286.959 & -145.342 & -88.511 & 56.482 & -53.696 & 153.361 & 1.0003 & $5.22 \times 10^{10}$ \\
\hline 1750 & -268.200 & 11.266 & -159.695 & -287.125 & -132.055 & -88.611 & 55.540 & -54.342 & 162.490 & 1.0002 & $5.29 \times 10^{10}$ \\
\hline
\end{tabular}

a) $\Delta H^{\ominus}: \mathrm{kJ} \cdot \mathrm{mol}^{-1}, \Delta G^{\ominus}: \mathrm{kJ} \cdot \mathrm{mol}^{-1}, \Delta S^{\ominus}: \mathrm{J} \cdot \mathrm{K}^{-1} \cdot \mathrm{mol}^{-1}, \Delta_{\mathrm{r}}^{\neq} H_{m}^{\ominus}: \mathrm{kJ} \cdot \mathrm{mol}^{-1}, \Delta_{\mathrm{r}}^{\ddagger} S_{\mathrm{m}}^{\ominus} \mathrm{J} \cdot \mathrm{K}^{-1} \cdot \mathrm{mol}^{-1}, \Delta_{\mathrm{r}}^{\neq} G_{\mathrm{m}}^{\ominus}: \mathrm{kJ} \cdot \mathrm{mol}^{-1}$ 

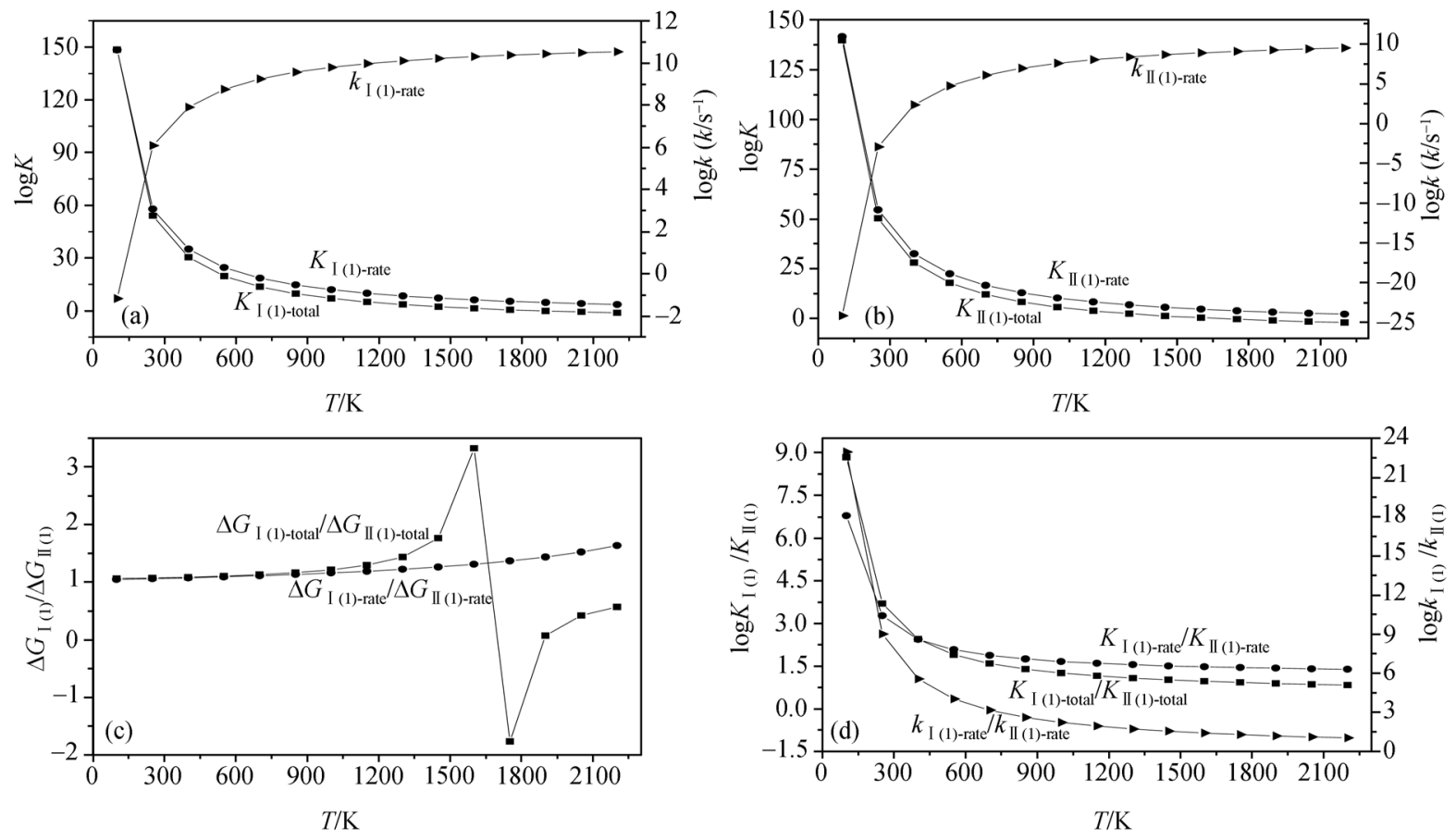

图 7 反应平衡常数 $K$, 速率常数 $k, K_{\left.\right|_{(1)}} / K_{\|(1)}, k_{\perp(1)} / k_{\|(1)}$ 的对数值及 $\Delta \mathrm{G}_{\mathrm{I}_{(1)}} / \Delta \mathrm{G}_{\|(1)}$ 随温度 $T$ 的变化曲线

的变化曲线. 从图可以看出, 随着温度的升高, 反应 I (1)及 II (1)的平衡常数 $K$ 均逐渐减小, 而速率常数 $k$ 逐渐增大, 当 $T$ 分别大于 $1750 \mathrm{~K}$ 和 $1600 \mathrm{~K}$ 时, $\mathrm{R} \rightarrow \mathrm{P} 1$ 和 $\mathrm{R} \rightarrow \mathrm{P} 4$ 的 $\log K<0$, 而当 $T<250 \mathrm{~K}$ 时, 反应 $\mathrm{IM} 1.1 \rightarrow$ $\mathrm{TS} 1.1 \rightarrow \mathrm{P} 1$ 和 $\mathrm{IM} 4.1 \rightarrow \mathrm{TS} 4.1 \rightarrow \mathrm{P} 4$ 的 $k$ 值也已很小, 表 明反应 I (1)和反应 II (1)在低温下具有热力学优势而 高温下具有动力学优势. 综合分析, 在 $101325 \mathrm{~Pa}$ 下, 反应 I (1)和 II (1)发生的适宜温度范围分别为 250 1750 和 $250 \sim 1600 \mathrm{~K}$, 如此条件下, 反应不但具有较 大的自发趋势和平衡转化率, 也具有较快的反应速 率.

图 7(c)为 $\Delta G_{\text {। (1)-total }} / \Delta G_{\text {॥(1)-total }}$ 及 $\Delta G_{\text {। (1)-rate }} / \Delta G_{\text {॥(1)-rate }}$ 与反应温度 $T$ 的关系图. 可以看出, 温度对 $\Delta G_{\text {। (1)-rate }} /$ $\Delta G_{\|(1)-\text { rate }}$ 及 $\Delta G_{\text {।(1)-total }} / \Delta G_{\| \text {(1)-total }}$ 的影响不大, 在所研 究的温度范围内, 在相同温度下, 反应 I (1)和 II (1) 的自发趋势并无显著性差异.

图 7(d) 为 $K_{\text {| (1)-rate }} / K_{\text {|| (1)-rate }}, K_{\text {| (1)-total }} / K_{\text {|| (1)-total }}$ 及

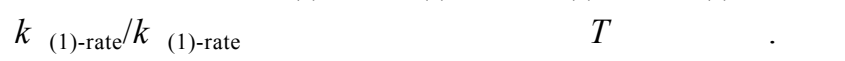
图可以看出, 温度对反应 I (1)及 II (1)的平衡常数 $K$ 及速率常数 $k$ 均有很大影响. 当温度为 $100 \mathrm{~K}$ 时,
$K_{\text {।(1)-rate }}, K_{\text {।(1)-total }}$ 和 $k_{\text {।(1)-rate }}$ 分别约为 $K_{\text {॥(1)-rate }}, K_{\text {||(1)-total }}$

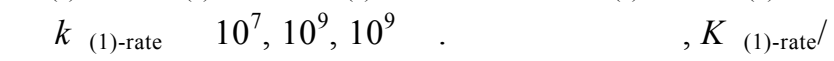
$K_{\text {॥ (1)-rate }}, K_{\text {।(1)-total }} / K_{\| \text {(1)-total }}$ 和 $k_{\text {। (1)-rate }} / k_{\|(1) \text {-rate }}$ 逐渐减小, 当温度 $T=1000 \mathrm{~K}$ 时, 其值均接近于 1 , 而当温度进 一步升高时, 其值几乎不再变化, 在此条件下, 对于 反应 II (1)来说, I (1)的反应速率已无优势可言.

综上分析, 在所研究的温度范围内, 生成 P1 的反 应 I (1)与生成 P4 的反应 II (1)相互竞争, 在低温下, 反应 I (1)与反应 II (1)的平衡转化率和反应速率相差 很大, 在保证反应 I (1)有足够大的反应速率的情况 下, 综合考虑, 在 250 1000 K 的温度范围内, 更有利 于单重态二溴卡宾插入乙醛 $\mathrm{C}_{\alpha}-\mathrm{H}$ 键生成产物 $\mathrm{P} 1$ 的 反应 I (1)的发生, 而在 $1000 \sim 1600 \mathrm{~K}$ 的温度范围内, 反应 I (1)及 II (1)没有明显的选择优势.

另外, 将卡宾及二溴卡宾与乙醛中 $\mathrm{C}-\mathrm{H}$ 键的插 入反应速率进行了对比. 在 $101325 \mathrm{~Pa}$ 和 $298.15 \mathrm{~K}$ 下, 卡宾与乙醛甲基氢的插入反应速率分别约为二溴卡 宾与乙醛中甲基氢及 $\mathrm{C}_{\alpha}-\mathrm{H}$ 插入反应速率的 $10^{11}$ 倍 和 $10^{5}$ 倍, 表明卡宾与乙醛中 $\mathrm{C}-\mathrm{H}$ 的插入反应更容 易发生, 这与图 2 和 4 的结论一致. 


\section{参考文献}

1 Houk K N, Rondan N G, Cielo S, et al. Theoretical studies of the structures and reactions of substituted carbonyl ylides. J Am Chem Soc, 1980, 102(5): 1504-1512[DOI]

2 Gill H S, Landgrebe J A. Selective formation and trapping of dihalocarbonyl ylides derived from dihalocarbenes and substituted benzaldenhydes. J Org Chem, 1983, 48(7): 1051-1055 $\underline{\text { [DOI] }}$

3 Díaz-Requejo M M, Belderraín T R, Nicasio M C, et al. Intermolecular copper-catalyzed carbon-hydrogen bond activation via carbene insertion. J Am Chem Soc, 2002, 124(6): 896-897 [DOI]

4 Hilt G, Galbiati F. Regioselective carbene insertion on polysubstituted dihydroaromatic compounds. Org Lett, 2006, 8(10): 2195$2198 \underline{\text { DOI] }}$

5 Seyferth D, Burlitch J M, Minasz R J, et al. Halomethyl-metal compounds. II. The preparation of gem-dihalocyclopropanes by the reaction of phenyl(trihalomethyl)mercury compounds with olefins. J Am Chem Soc, 1965, 87(19): 4259—4270

6 Seyferth D, Tronich W. Reactions of phenyl(bromodichloromethyl)mercury. Preparation of perchlorothiirane. J Am Chem Soc, 1969, 91(8): 2138-2139

7 Peng L, Li Q S, Fang W H, et al. Theoretical study on cycloaddition of singlet dichlorocarbene with formaldehyde, acetaldehyde and benzaldehyde and subsequent rearrangement reactions. Chem Phys Lett, 2003, 382(1): 126-132 [DOI]

8 Morilla M E, Molina M J, Díaz-Requejo M M, et al. Copper-catalyzed carbene insertion into O-H bonds: High selective conversion of alcohols into ethers. Organometallics, 2003, 22(14): 2914-2918[DOI]

9 Fang R, Geng Z Y, Wang Y C, et al. Theoretical study of the insertion reaction of $\mathrm{CH}_{3} \mathrm{O}-\mathrm{H}$ by singlet alkylidenecarbenes. Chem Phys Lett, 2005, 414(1-3): 174-179 [DOI]

10 Gordon M S, Gano D R. Ab initio study of the insertions of methylene and silylene into methane, silane, and hydrogen. J Am Chem Soc, 1984, 106(19): 5421-5425[DOI]

11 Bach R D, Su M D, Aldabbagh E, et al. A theoretical model for the orientation of carbene insertion into saturated hydrocarbons and the origin of the activation barrier. J Am Chem Soc, 1993, 115(22): 10237-10246[DOD]

12 林启君, 冯大诚, 马万勇, 等. 卡宾与醚 $\mathrm{C}-\mathrm{H}$ 键插入反应的理论研究( II) 一二氯卡宾和二氟卡宾与二甲醚的插入反应. 高 等学校化学学报, 2000, 21(9): 1427-1431

13 冯大诚, 林启君, 马万勇, 等. 卡宾与醚 $\mathrm{C}-\mathrm{H}$ 键插入反应的理论研究 (III) - $\mathrm{CX}_{2}(\mathrm{X}=\mathrm{H}, \mathrm{F}, \mathrm{Cl})$ 与甲 乙醚 $\mathrm{C}-\mathrm{H}$ 键插入反应. 高 等学校化学学报, 2000, 21(11): 1708-1712

14 林启君, 冯大诚, 戚传松. 卡宾与醚 $\mathrm{C}-\mathrm{H}$ 键插入反应的理论研究 ( IV $)-\mathrm{CX}_{2}(\mathrm{X}=\mathrm{H}, \mathrm{F}, \mathrm{Cl})$ 与甲基芐基醚 $\mathrm{C}-\mathrm{H}$ 键插入反应. 高 等学校化学学报, 2000, 21(12): 1922-1924

15 卢秀慧, 武卫荣, 于海涁, 等. 二甲基亚烷基卡宾与丙酮环加成反应机理的理论研究. 科学通报, 2005, 50(16): 1693-1699

16 耿志远, 姚琨, 王永成, 等. 亚烷基锗烯与环氧乙烷及环硫乙烷抽提氧和硫反应的量子化学研究. 中国科学 B 辑: 化学, 2007, 37(2): $127-135$

$\mathrm{Xu}$ Z F, Lin M C. Computational studies on the kinetics and mechanisms for $\mathrm{NH}_{3}$ reactions with $\mathrm{ClO}_{x}(x=0-4)$ radicals. J Phys Chem A, 2007, 114(4): 584-590[DOI]

Chen X Y, Zhao C X, Ping Y, et al. Theoretical study of reactions between $\mathrm{MH}(\mathrm{M}=\mathrm{B}, \mathrm{Al})$ and the $\mathrm{H}_{2} \mathrm{~S}$ molecule. Int J Quant Chem, 2001, 85(3): 127-135[DOI]

Kuninobu Y, Nishina Y, Nakagawa C, et al. Rhenium-catalyzed insertion of aldehyde into a C-H bond: Synthesis of isobenzofuran derivatives. J Am Chem Soc, 2006: 128 (38): 12376-12377 [DOI]

20 李志锋, 吕玲玲, 康敬万, 等. $\mathrm{CBr}_{2}$ 与 $\mathrm{CH}_{2} \mathrm{O}$ 插入反应机理的密度泛函理论研究. 科学通报, 2007, 52(8): 880-885

21 Su M D, Chu S Y. Density functional study of some germylene insertion reactions. J Chem Soc, 1999, 121(17): 4229—4237[DOI]

Kollmar H. Insertion reaction of a nucleophilic carbene. A molecular orbital theoretical study. J Am Chem Soc, 1978, 100(9): $2660-2664$

Jeziorek D, Zurawski B. Ab initio calculations including electron correlation for the minimum energy path of the $\left({ }^{1} \mathrm{~A}_{1}\right) \mathrm{CH}_{2}+\left({ }^{1} \Sigma_{\mathrm{g}}{ }^{+}\right) \mathrm{H}_{2}$ $\rightarrow\left({ }^{1} \mathrm{~A}_{1}\right) \mathrm{CH}_{4}$ insertion reaction. Int J Quant Chem, 1979, 16(2): 277-297

Reed A E, Weinstock R B, Weinhold F. Natural population analysis. J Chem Phys, 1985, 83(2): 735 — 746[DOI]

Reed A E, Weinhold F. Natural localized molecular orbitals. J Chem Phys, 1985, 83(4): 1736-1740[DOI] 